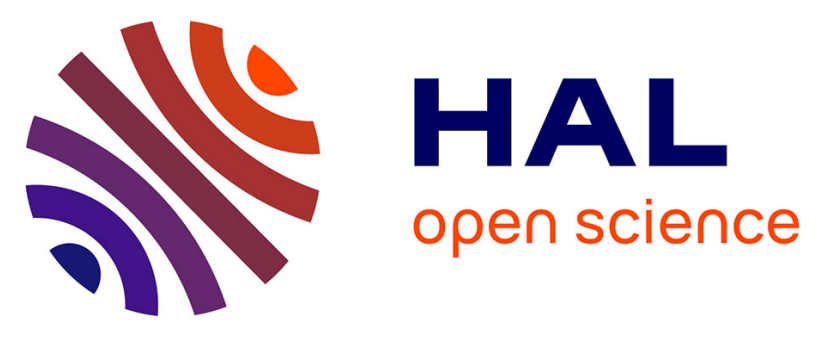

\title{
Understanding hierarchy and functions of bone using scanning x-ray scattering methods
}

\author{
Wolfgang Wagermaier, Aurélien Gourrier, Barbara Aichmayer
}

\section{To cite this version:}

Wolfgang Wagermaier, Aurélien Gourrier, Barbara Aichmayer. Understanding hierarchy and functions of bone using scanning x-ray scattering methods. Peter Fratzl, John W.C. Dunlop, Richard Weinkamer. Understanding hierarchy and functions of bone using scanning x-ray scattering methods, RSC publishing, 2013, From the book Materials Design Inspired by Nature: Function Through Inner Architecture, 978-1-84973-553-7. 10.1039/9781849737555-00046 . hal-01388704

\section{HAL Id: hal-01388704 https://hal.science/hal-01388704}

Submitted on 27 Oct 2016

HAL is a multi-disciplinary open access archive for the deposit and dissemination of scientific research documents, whether they are published or not. The documents may come from teaching and research institutions in France or abroad, or from public or private research centers.
L'archive ouverte pluridisciplinaire HAL, est destinée au dépôt et à la diffusion de documents scientifiques de niveau recherche, publiés ou non, émanant des établissements d'enseignement et de recherche français ou étrangers, des laboratoires publics ou privés.

\section{(1) $\$(0)$}

Distributed under a Creative Commons Attribution - NonCommercial - ShareAlikel 4.0 


\title{
Chapter 3
}

\section{Understanding hierarchy and functions of bone using scanning $x$-ray scattering methods.}

\author{
Wolfgang Wagermaier' ${ }^{1}$, Aurelien Gourrier ${ }^{2,3}$, Barbara Aichmayer ${ }^{1}$ \\ ${ }^{1}$ Department of Biomaterials, Max Planck Institute of Colloids and Interfaces, Potsdam, Germany \\ ${ }^{2}$ Laboratoire Interdisciplinaire de Physique, Université Joseph Fourier, Grenoble, France \\ ${ }^{3}$ European Synchrotron Radiation Facility, Grenoble, France
}

\section{Table of contents}

\section{Abstract.}

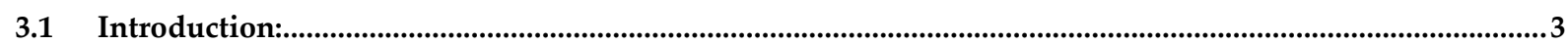

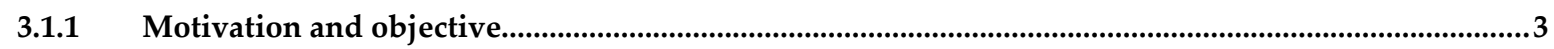

3.1.2 X-ray scattering applied to the study of biological materials.............................................................4

3.1.3 Bone as a model for a hierarchically structured material..........................................................................

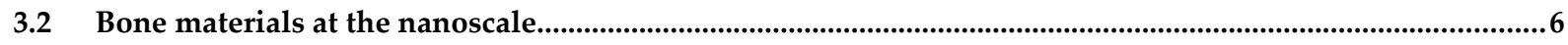

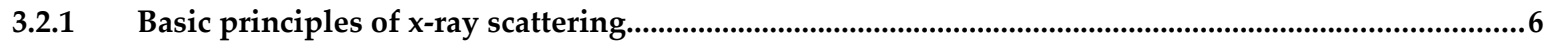

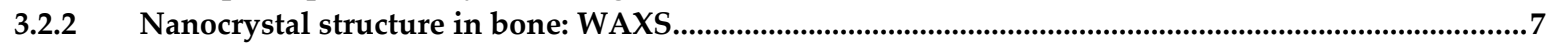

3.2.3 Mineral particle size and organization in the collagen matrix: SAXS.................................................11

3.2.4 SAXS and WAXS of precursor phases found in bone................................................................14

3.3 Understanding specific bone functions by investigating the nanostructure in combination with other methods.

Multi-scale and multi-physics approach

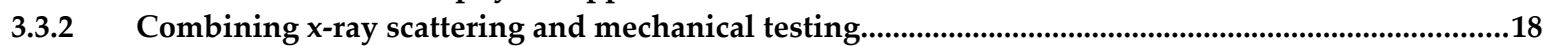

3.4 Revealing the nanoscale properties of bone tissues and organs: scanning SAXS/WAXS imaging.............20

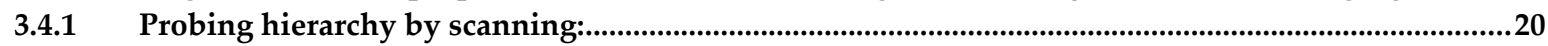

3.4.1.1 Instrumental aspects of scanning experiments using $X$-ray microbeams.................................20

3.4.1.2 Qualitative versus quantitative sSAXS/WAXS imaging...............................................................22

3.4.1.3 Image resolution versus field of view..................................................................................................24

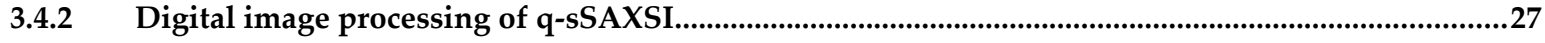

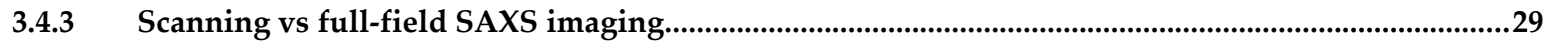

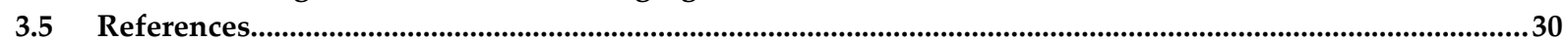




\begin{abstract}
Biological materials are often hierarchically structured from the nanometer to the macroscopic scale. Specific characterization methods are needed to characterize the structures at these different length scales. This chapter reviews -based on the example of bone- the use of X-ray scattering methods to explore representative and quantitative structure information as well as structure-function relations in hierarchically structured biological materials. X-ray scattering techniques are particularly well suited for the characterization of the form and organization of organic and inorganic components in those materials. When nanometer-sized structures are exposed to X-rays, details of the internal material structure can be revealed by the analysis of the resulting interference patterns. Fundamental aspects of wide and small angle X-ray scattering (WAXS and SAXS) are discussed with specific focus on bone studies. An important field of research using X-ray scattering techniques, is the in situ combination with mechanical testing, which allows investigating changes in structure under specific loading conditions. Another common application is the structural study of heterogeneities or local structures within a sample using a narrow focused X-ray beam. Furthermore, in scanning mode, where the specimen is displaced step by step across a microbeam while collecting a SAXS/WAXS pattern at each step, complex structural maps of the sample can be derived. A natural extension of the method toward imaging is described in the context of X-ray imaging with scattering contrast.
\end{abstract}




\subsection{Introduction}

\subsubsection{Motivation and objective}

Many biological and bio-inspired synthetic materials are hierarchically structured and show a composite character at the nanoscale [1]. This characteristic pattern of well identified structural elements imbricated at different length scales determines the properties of the material. Understanding structure - function relations and unraveling in detail the complex structural features of biological materials are important goals for research in biomechanics and structural biology [2, 3]. Biological materials science aims to understand these hierarchical structures by using methods ranging from materials physics and chemistry to biology and medicine. One single method is usually not sufficient to answer such complex issues and therefore several techniques are often combined to explore the structures at different size levels.

Biological and bio-inspired materials are produced adopting a bottom-up approach. Thus, the basic building blocks can only be revealed using analytical techniques with nanoscale sensitivity. X-ray scattering methods provide, in particular, quantitative information on the arrangement of atoms in crystals, molecules and supramolecular entities in biomaterials at the length scale of $0.1 \mathrm{~nm}-100 \mathrm{~nm}$ $[4,5]$. This chapter describes how X-ray scattering methods can be applied to biological and bioinspired materials to answer a variety of questions. Based on the example of bone, different approaches are presented to gain insight into the organization at different hierarchical levels. Following an introduction to basic principles of X-ray scattering, illustrated with several examples from bone studies (section 2), the next section describes how the nanostructure can be investigated in relation to its function (section 3). The final section (4) shows how X-ray scattering methods can be extended to visualize and quantify higher length scale structures through micro-imaging approaches.

\subsubsection{X-ray scattering applied to the study of biological materials}

Due to their high level of structural hierarchy, biological and bio-inspired materials are generally investigated using a combination of imaging methods. Structures at the millimeter level and down to several hundred of nanometers (corresponding to the wavelength of light) can be explored using different light microscopy techniques. Structural elements on the micrometer and sub-micrometer level are often examined by scanning electron microscopy, while, at the nanometer level, more refined techniques are required, such as transmission electron microscopy (TEM) or atomic force microscopy (AFM), However, in order to probe the fine details of the atomic and nanoscale structure, the analysis is very often complemented by X-ray scattering methods. If the structural units exposed to X-rays are ordered at the nanoscale or below, the elastically scattered radiation gives rise to diffraction and other interference effects in the wide-angle X-ray scattering (WAXS) regime [4, 6] as well as in the smallangle X-ray scattering (SAXS) regime [7, 8]. Thus, an analysis of the patterns recorded on a 2D detector delivers quantitative values of structural parameters of interest averaged over the illuminated sample volume. Besides its quantitative nature, X-ray scattering has also the advantage that the method requires only simple sample preparation. One drawback, however, is the need for complex structural models to deduce a detailed description of the sample based on its scattering pattern in reciprocal space. Nevertheless, a basic set of generic physical parameters which are relevant to biomineral studies in particular and two-phased media in general, can be calculated as will be described in this chapter.

\subsubsection{Bone as a model for a hierarchically structured material}

Bone is a good example of a hierarchically structured biomaterial [9]. Hierarchical levels in bone range from the basic building block at the molecular level - the mineralized collagen microfibril [10, 11] (nmrange: molecular level) - over their staggered arrangement into fibrils ( $\mu \mathrm{m}$-range: material level) up to the assembly into fibers that form structures like osteons ( $\mu \mathrm{m}$ and mm-range: tissue level). The highest hierarchical levels include cortical or spongy bone and finally the overall geometry of whole bones ( $\mathrm{mm}$ and cm-range: organ level). The diversity of bone structures [12] is believed to reflect the continuous adaptation of the structure to adequately address the requirements of specific biological functions $[9,13]$. The fact that bone is inherently hierarchical in its architecture and heterogeneous due 
to its composite nature makes it an ideal model to describe the use of X-ray scattering methods to clarify highly relevant questions from different fields. In order to extract detailed structural information at the nanometer level in bone, it is crucial to use quantitative, nondestructive techniques such as X-ray diffraction and scattering. Figure 1 shows some selected hierarchical levels of bone and a set of corresponding methods which can be used to investigate the structural features as well as the related functions. At the atomic and nanometer level WAXS and SAXS can be used to determine the crystalline arrangement of the mineral particle, their size, orientation and organization. By scanning over the sample with the X-ray beam the method can also be extended to higher length scales to image features at the micro- and even millimeter level.

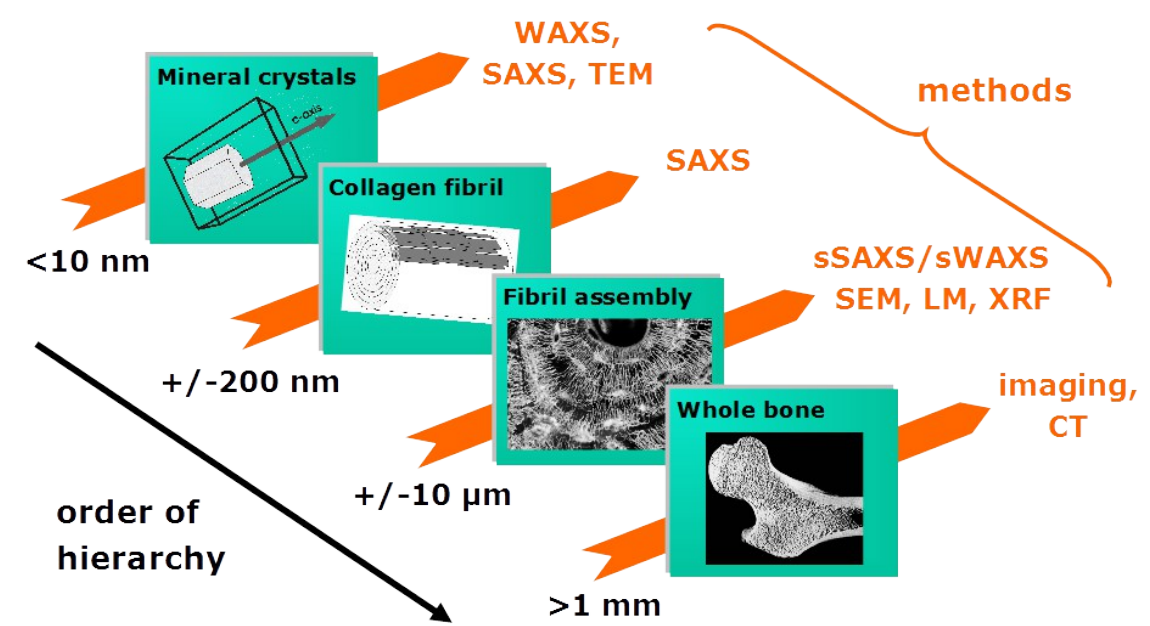

Figure 1: Four different hierarchical levels in bone and some methods to investigate the material structure at these different length scales: SAXS, WAXS: small and wide angle x-ray scattering, TEM: transmission electron microscopy, sSAXS/sWAXS: scanning SAXS/WAXS, SEM: scanning electron microscopy, LM: light microscopy, XRF: x-ray fluorescence, CT: micro computed tomography

\subsection{Bone materials at the nanoscale}

\subsubsection{Basic principles of $x$-ray scattering}

$\mathrm{X}$-ray scattering is based on the interaction of electromagnetic waves (or photons) with matter. More specifically, X-rays with wavelengths from 0.01 to $10 \mathrm{~nm}$, interact with electrons. Hence, X-ray scattering is used to probe the distribution of the electron density in the material [14].

Structural investigations by X-ray scattering are mainly based on elastic scattering, i.e. without any change of energy/wavelength between the incoming and measured radiation. While the term scattering implies a general deviation of waves/photons within the traversed medium, diffraction is more specific and stands for an interaction with highly ordered structures resulting in interference effects of waves/photons in specific directions. The most fundamental parameter to consider in scanning X-ray scattering studies is the scattering power of the sample. This is an intrinsic property of materials at a given wavelength. The X-ray scattering power essentially depends on the number of electrons in the atoms, i.e. it increases for heavier atoms.

The scheme of the setup of an X-ray scattering experiment is shown in figure 2a. A fraction of the incident beam (wavelength $\lambda$ ) is scattered by the sample while most of the remaining part is transmitted through without interacting. The measured intensity of the scattered beam $I(\vec{q})$ is related to the square of the Fourier transform of the scattering length density distribution. The scattering vector $\vec{q}$ is defined as the difference between the wave-vector of the incident and the scattered beam (figure $2 \mathrm{~b}$ ). During the experiment the scattered intensity is monitored as a function of the scattering angle $2 \theta$.

$$
\left.|\vec{q}|=q=\frac{4 \pi}{\lambda} \sin \theta \quad \text { (equation } 1\right)
$$




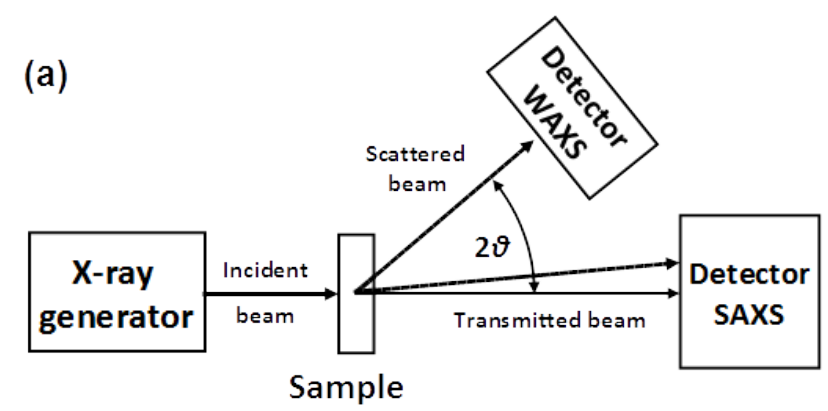

(b)
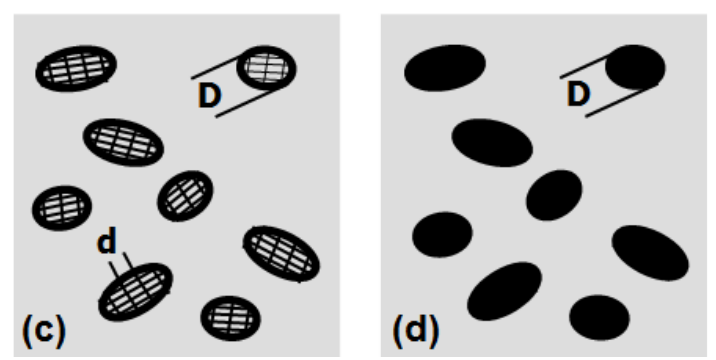

SAXS: particle size (D) and orientation

WAXS: crystal lattice parameters (d) and crystal size

Figure 2: (a) $x$-ray scattering setup, (b) geometry defining the scattering dector between incident and scattered beam, (c) crystalline particles in a matrix, (d) non-crystalline particles in a matrix.

On the basis of a critical angle (typically $2 \theta \sim 5^{\circ}$ ) a separation is usually made between small-angle Xray scattering (SAXS) and wide-angle X-ray scattering (WAXS). In the wide angle range the so called Bragg diffraction provides information on crystal parameters, while SAXS can be used to determine structural parameters in more loosely ordered systems (see figure $2 \mathrm{c}$ and $2 \mathrm{~d}$ ). Although this distinction is somewhat arbitrary, since very large crystal lattice parameters can also give rise to diffraction in the SAXS region, it is, nevertheless, verified in a large number of cases, including most biological materials.
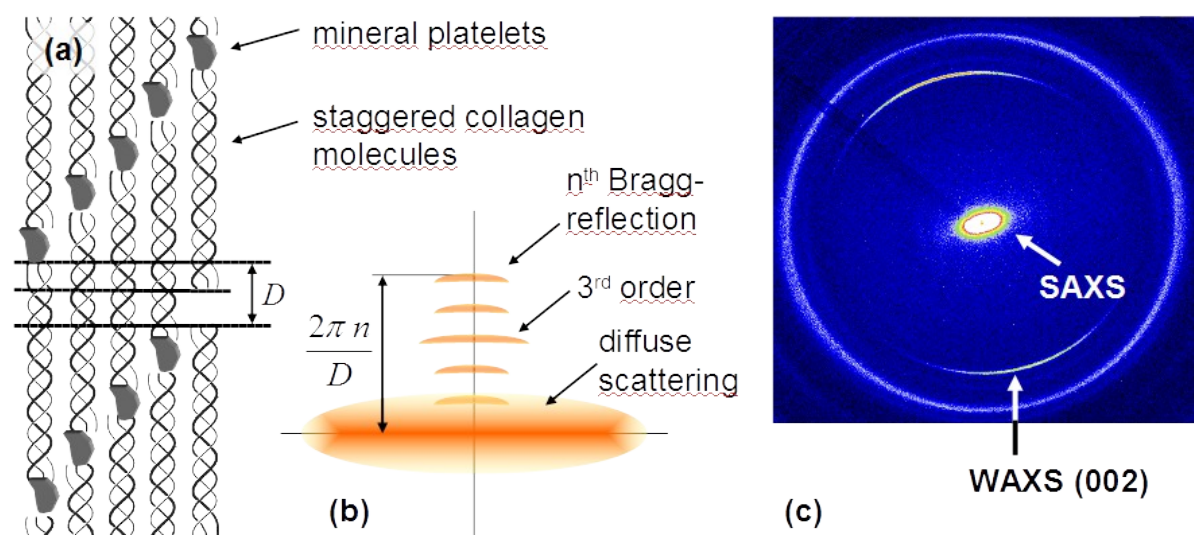

Figure 3: (a) Arrangement of collagen-fibrils (after Hodge-Petruska, 1964) and definition of the D-period, (b) schematic scattering and diffraction pattern, (c) combined SAXS/WAXS signal measured with a two-dimensional CCD.

In the case of X-ray scattering from a bone sample, the interpretation of SAXS and WAXS signals can be described as follows (see figure 3). The typical small-angle X-ray scattering pattern from bone consists of a diffuse anisotropic signal originating from the thin mineral platelets (with high specific surface area), and a series of small-angle X-ray diffraction Bragg peaks, arising from the axial periodicity in the arrangement of the intrafibrillar collagen molecules [15] (figure 3a and 3b). The axial stagger is characterized by a period $D$ which can range from 64 to $67 \mathrm{~nm}[8,16]$. Due to the difference in electron density between the mineral and organic phases, the diffuse signal from the mineral platelets is much stronger than this of the collagen fibrils, such that the latter is often not detected. In the wide angle regime, the mineral particles of carbonated apatite in bone give rise to diffraction peaks. Apatite has a hexagonal cubic lattice structure, with the (002) or c-axis oriented along the 
collagen fibril axis. Hence, the fibril orientation can be obtained from the (002) lattice spacing of the apatite WAXS pattern (figure 3c). A more detailed description of WAXS analysis and its application to bone is given in the following section.

\subsubsection{Nanocrystal structure in bone: WAXS}

Wide-angle X-ray scattering reveals the nature of crystalline materials, i.e. if atoms in the investigated material are regularly ordered. A periodic three-dimensional arrangement of atoms or molecules acts as a diffraction grating, where the electron cloud of each atom is excited by the incoming X-rays and becomes a secondary source of radiation. Diffraction from a perfect single crystal would result in a characteristic pattern (for this crystal) on a two-dimensional detector. If the exposed material is polycrystalline the spots on the detector turn into rings (Debye-Scherrer rings), since the different crystal orientations result in different orientations of the scattered beam along a cone with an opening angle of $2 \theta$.

From a diffractogram (plot of the azimuthally averaged intensity over the scattering vector $q$ ), different parameters can be determined: (i) the peak position gives information on the lattice spacing, (ii) the area of the peak is a relative measure for the amount of scattered elements (degree of crystallinity) and (iii) the peak width gives information on crystal sizes.

\subsubsection{Bragg's law - determining lattice spacings}

Bragg's law is a relatively simple relationship between crystallite structure and parameters as determined from $x$-ray scattering [17]:

$$
n \lambda=2 d \sin (\theta) \quad \text { (equation 2) }
$$

Here, $d$ is the distance between planes in the atomic lattice and $n$ is an integer given by the order of the reflection. By using Bragg's law typical distances in the material can be calculated from the position of peaks in a scattering curve, which is a plot of the measured intensity $I$ as a function of $q$ or the angle $2 \theta$. Sharp Bragg peaks can be observed in the diffraction pattern if atoms are perfectly ordered in a crystalline structure. Peaks in the SAXS regime indicate a long period between areas with similar electron density if they appear repeatedly. If the system is not perfectly ordered, as is typical in the case of soft matter, such as the organic phase in bone, the intensity of the Bragg peaks is reduced, the width of the peaks is larger and the intensity scattered by the most disordered part appears as a diffuse background.

\subsubsection{Scherrer equation - determining crystal sizes}

From the widths of crystalline reflections an average crystal size can be determined by use of the Scherrer equation $[18,19]$ :

$$
L_{h k l}=\frac{K \cdot \lambda}{\beta \cdot \cos \theta}
$$

(equation 3)

In this equation, $L_{h k l}$ is the crystallite size perpendicular to the $(h k l)$ plane ( $h k l$ are the Miller indices, defining the orientation of an atomic plane), $K$ is the Scherrer constant depending on the crystallite shape, $\lambda$ is the wavelength of radiation, and $\beta$ the integral width of the peak (in radians $2 \theta$ ) located at an angle $\theta$. Furthermore, by determining shifts in the peak position, strains within the crystals can be detected [20].

\subsubsection{Texture - preferred orientations}

Texture is the spatial orientation distribution of crystallites in a polycrystalline material and is a property of crystalline solids [21]. The crystalline structure has a major impact on the mechanical, physical, chemical and technological properties on the microscopic scale (single crystal aggregates) as well as on the macroscopic scale of materials. Anisotropic properties of single crystallites distributed randomly in a polycrystalline material would result in an isotropic behavior [22]. In bone, like in many materials the crystals are not fully randomly distributed and therefore a preferred orientation of crystals results in anisotropic behavior, which can be described by its texture [6]. 


\subsubsection{WAXS analysis of bone material:}

As biological materials are often composites made of an organic and a mineral phase, $X$-ray diffraction in those cases mainly provides information from the crystalline mineral particles. The lattice of the mineral particles in bone (carbonated hydroxyapatite) can be described by hexagonal crystal symmetry. Different Debye-Scherrer rings of bone show diverse intensities, most of which are quite poor [6]. The strongest distinct ring in bone corresponds to the (002) lattice planes followed by the (310) lattice planes. The position of the (002)-peak in a diffractogram of bone describes the lateral spacing along the crystallographic c-axis in the HA. The evaluation of the peak width following the Scherrer equation can be used to describe the length of the crystalline particles (often called $L$ parameter) [20].

The orientation of the hexagonal c-axis of the mineral particles can be determined by quantitative texture analysis with X-rays [4, 23-25] or by neutron diffraction measurements [26]. The probability that a HA c-axis is oriented in a particular direction is given by the intensity distribution along the Debye Scherrer rings. The (002) reflection in turn allows the determination of the collagen fibril orientation since there is a strong correlation between these orientations. In different studies, it was shown that the hexagonal c-axis of the plate-shaped mineral particles points preferentially into the fibrillar direction $[2,11,27]$. Using scanning X-ray scattering with a micron-sized synchrotron beam and analyzing the local mineral crystallographic axis directions, the three-dimensional orientation of mineralized fibrils within single osteon lamella (around $5 \mu \mathrm{m}$ ) could be reconstructed [28, 29]. By this method it could be shown that the mineralized collagen fibrils spiral around the central axis with varying degrees of tilt, which would impart high extensibility to the osteon. In addition to the quantification of the mineral orientation itself, this fact enables to investigate the arrangement of the entire fibrils in which the mineral particles are embedded.

\subsubsection{Mineral particle size and organization in the collagen matrix: SAXS}

SAXS provides a measure of changes in electron density in a material and, consequently, it enables to determine quantitative information on the size and orientations of particles at the nanometer scale (see figure $2 \mathrm{c}$ and $2 \mathrm{~d}$ ). The SAXS pattern of any material containing heterogeneities at the nanoscale is usually described as the product of a form factor, $F(q)$, with a structure factor, $S(q)$. The former relates to the shape and size of the nanometer-sized features, while the second depends on their long-range arrangement. In the case of bone, numerous TEM studies have indicated that the mineral phase is found in the form of elongated platelets of approximate thickness, width and height: $5 \times 25 \times 50 \mathrm{~nm}^{3}$ $[2,30-34]$. Interestingly, it seems that the most regular dimension for a wide range of species and anatomical sites is the particle thickness.

\subsubsection{The size of mineral particles: T-parameter}

A basic assumption in the presented approach is a system consisting of two phases with different average electron density and with sharp interfaces between them [35-37]. According to Porod's law, for such a two-phase system the scattering intensity at large q decreases with $\mathrm{q}^{4}$ (figure $4 \mathrm{a}$ and $4 \mathrm{~b}$ ):

$$
\left.I(q \rightarrow \infty) \approx B+\frac{P}{q^{4}} \quad \text { (equation } 4\right)
$$

$B$ is a background resulting from incoherent and inelastic scattering and $P$ is the Porod constant which can be calculated from the Porod-plot (figure 4c).

In bone, these phases comprise the mineral and organic components. Information on the mean mineral particle thickness in bone (also called "T-parameter") can be derived from the decay of the scattering signal (at large $q$ ) and the total integrated scattered intensity [38-40]. The T-parameter is calculated using the following equation:

$$
T=\frac{4}{\pi \cdot P} \int_{0}^{\infty} q^{2} I(\vec{q}) d q=4 \frac{\phi(1-\phi)}{\sigma}
$$


where $\phi$ is the volume fraction of the mineral phase and $\sigma$ the surface area of mineral particles per unit volume and. The integral value $J\left(J=\int I(q, \chi) q^{2} d q d \chi\right)$ is often referred to as the invariant in the theory of SAXS since, for spherically isotropic system, this quantity depends only on the volume fraction of the two homogeneous phases, independently of their distribution. However, since in bone the mineral particles are strongly anisotropic, the particle orientation strongly influences this value [41] which will be termed integrated SAXS intensity thereafter. Assuming that the mineral phase has a shape of a uniform parallelepiped (edge lengths $a, b$ and $c$ ) the equation can be modified to

$$
T=2(1-\phi) \frac{a b c}{a b+b c+a c}
$$

In bone the mineral particles are assumed to be platelets with one significant shorter dimension than the others $(\mathrm{a}<<\mathrm{b}, \mathrm{c})$ [2]. With this, the equation simplifies to $T \approx 2(1-\phi) a$. Therefore the Tparameter gives directly the mineral particle thickness only if the degree of mineralization $\phi$ corresponds to 0.5 [42]. In order to calculate the T-parameter, $\sigma$ and $\phi$ have to be determined. The integral in equation 5 can be solved by determining the area A under the function $I(q) \cdot q^{2}$ (Kratky-Plot, figure $4 \mathrm{~d}$ ) from $\mathrm{q}=0$ to $\mathrm{q}=\infty$. This procedure is described in more detail in [40].
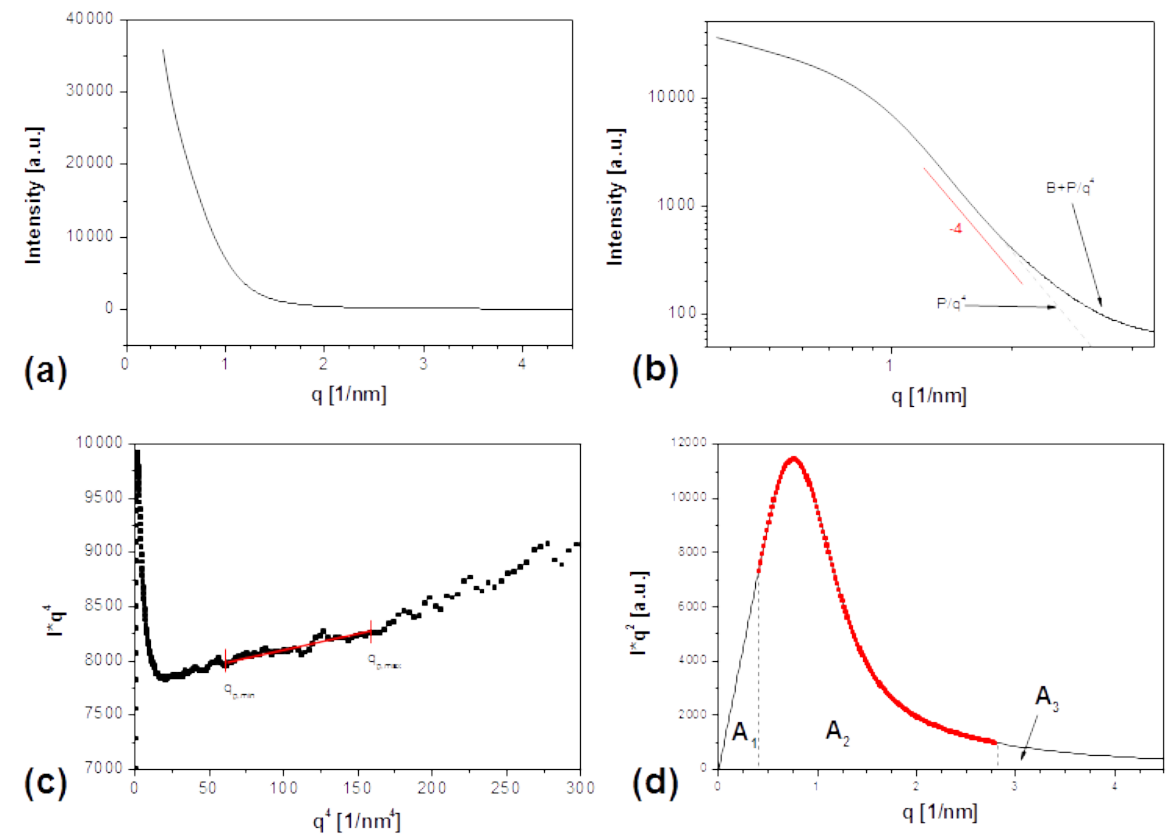

Figure 4: Scattering intensity as a function of the scattering vector: (a) I vs. q, (b) log log plot of I vs. q, (c) Porod plot, (d) Kratky plot.

\subsubsection{Lateral registration - stack of cards model}

The particles in bone have been observed to pack in a regular fashion along the transverse direction, similar to a stack of cards, with their long axis oriented along the fibril direction. Thus, SAXS patterns of bones should reflect this information. It turns out that this is only partly the case since the SAXS signal is fully described by the sole form factor, which is a prolate ellipsoid (i.e. the Fourier transform of a platelet) [43]. The absence of a structure factor therefore implies that the mineral platelet stacking is too irregular and doesn't extend to a long range order.

Recently, a relatively simple model was proposed to take into account possible order at short-range using a correlation function of the form: $g(\xi) \approx \mathrm{e}^{-\alpha \zeta} \cos (\beta \zeta)$, where $\beta$, contained in the oscillatory term, provides a measure of the regularity in inter-particle distance and $\alpha$ controls the damping by the decreasing exponential, to allow for the limited extent of the ordering [44]. This correlation function is related to the azimuthally integrated SAXS signal by a Fourier transform, such that, in practice, the use of this model involves fitting the curve $q^{2} I(q)$ with the following function: 


$$
q^{2} I(q)=P T^{2} \frac{q^{2} T^{2}+(\alpha-1)(\alpha+\beta)^{2}}{\left(q^{2} T^{2}+\alpha^{2}-\beta^{2}\right)^{2}+4 \alpha^{2} \beta^{2}}
$$

This model was recently applied to a clinical case of fluorosis and clearly allowed distinguishing pathological regions of bone with abnormal organization of the mineral particles from healthy ones, as evidenced by different values of $\alpha$ and $\beta$ [45]. Other models have been proposed using similar concepts $[46,47]$ and it could be anticipated that this short-range range ordering aspect, which hasn't received much attention so far in SAXS studies, could become of importance in future studies of bone.

\subsubsection{The orientation of mineral particles}

Based on the same assumptions as presented in the previous subsection (material with phases of different electron density at the nanoscale) the SAXS pattern can be used to determine the orientation and a degree of alignment of these structures. In the case of bone, the orientation and degree of alignment of mineral particles can be obtained $[2,39,40]$. The SAXS pattern would show a narrow streak perpendicular to the orientation of the long axis of the mineral particles, if the mineral particle alignment was perfectly parallel. In contrast, a spherically shaped SAXS pattern would appear if the particles were aligned fully randomly. Any other average particle alignment would cause elliptically shaped SAXS patterns. Therefore a predominant orientation of mineral particles can be estimated from the anisotropy of the elliptical SAXS pattern by computing $I(\chi)$, where $\chi$ is the azimuthal angle in the plane of the scattering pattern. From elliptical patterns, $I(\chi)$ shows two peaks (area A1) which are separated by $180^{\circ}$ on top of a constant background (area A0). This can be used to determine the degree of alignment. The total area under the curve $I(\chi)$ for $0<\mathrm{c}<360^{\circ}$ is $\mathrm{A} 1+\mathrm{A} 0$. The ratio $\mathrm{A} 1 /(\mathrm{A} 0+$ A1) is defined as the degree of alignment of the mineral particles, often called rho-parameter, revealing the fraction of non-isotropically aligned mineral particles [40]. The position of the peaks shows directly the predominant orientation of the mineral particles (rotated by $90^{\circ}$ ).

The evaluation of the rho-parameter has been used for example in a study to explore the changes in orientation of mineral particles between fetal and postnatal mouse bone tissue [48] as well as in a study on how porous scaffold architecture guides bone formation in a sheep model [49]. The rhoparameter describes the projected orientation of the particles within the exposed volume of the sample and does not give any information about the orientation outside of the plane. By tilting the sample with respect to the incoming beam and repeating the measurement for various tilt angles this threedimensional information can be obtained [50,51].

\subsubsection{SAXS and WAXS of precursor phases found in bone}

In addition to the characterization of mature bone (analysis described in chapters 2.2 and 2.3), scanning SAXS and WAXS were previously also used to investigate precursor phases in the early stages of bone formation. The structure of potential precursors in mineralized biological tissues might substantially deviate from their mature form. It is well known, that many biogenic calcium carbonate minerals are formed via the crystallization of a transient, amorphous precursor [52]. A similar mechanism, i.e. the formation of carbonated HA particles via transient precursor phases, was also suggested for the formation of bone. Experimental evidence was provided by mapping the formation of bone in zebrafish fin rays using SAXS/WAXS [53]. On the level of the mineralized collagen fibrils, the bony rays found in zebrafish fins have the same structure as all known bone materials. Since the bony rays continuously grow (in length as well as in diameter), scanning SAXS/WAXS allows mapping both, mature and forming regions of the bone. The core corresponds to mature bone and the tip as well as the lateral edges of the ray correspond to forming bone. Two concepts were used to analyze the scattering data of forming bone in zebrafish fins: the distinction between crystalline and amorphous mineral phases based on WAXS peaks and the comparison between the measured SAXS signals and the calculated scattering function of spheres. 


\subsubsection{WAXS of crystalline and amorphous phases}

Crystalline materials such as HA can be identified by the position $(q$ or $2 \theta)$ of their Bragg diffraction peaks (see 2.1). Besides instrumental broadening effects, any deviation from perfect ordering leads to a broadening of the diffraction peaks. Not only the finite size of the crystals (see 2.2, L-parameter), but also microstrain fluctuations can contribute to the peak width observed for biominerals [54]. For the extreme example of disordered (amorphous) mineral phases, one still observes peaks in the wideangle regime, which are however, substantially broadened. Whereas the peak position reflects the distance of the short range order found in the "amorphous" mineral phase, the peak area is proportional to the amount of the phase.

\subsubsection{SAXS of isolated particles - form factor}

For spherical particles, the scattering intensity corresponds to the following form factor:

$$
F_{s}^{2}(q)=\left(3 \frac{\sin (q R)-q R \cos (q R)}{(q R)^{3}}\right)^{2} \quad \text { (equation 8) }
$$

Form factors for other shapes can be found in ref. [55]. If the investigated sample consists of isolated particles (diluted system), the scattering contributions of all particles sum up incoherently and the total measured scattering intensity corresponds to the form factor multiplied by a constant factor. The constant factor depends on the instrumental setup, the square of the volume of the particles, the number of particles and the contrast between the particles and their surrounding. For non-diluted systems, the form factor needs to be multiplied by the structure factor, taking into account the interference between the scattering contributions of the particles. This mainly affects the scattering intensities at low q values (see 2.3, stack-of-cards model). For monodisperse spheres (narrow size distribution), the form factor at higher Q-values shows characteristic oscillations (see the example in figure 5). Such oscillations are also found for other shapes like cylinders and plates, as long as the particles are monodisperse. The position of these oscillations depends on the size of the particles and can hence be used to estimate their radius.

\subsubsection{SAXS/WAXS Results of a Zebrafish Fin}

Microbeam scanning of bony rays in a zebrafish fin reveals two distinct regions with characteristic SAXS and WAXS patterns (figure $5 \mathrm{a}$ and $5 \mathrm{~b}$ ). The WAXS patterns of the mature region (core of the bone) are consistent with HA (figure $5 \mathrm{c}$ ) and the T-parameters evaluated from SAXS curves of the same region are in the range of 2.2-2.5 nm [53], which is in agreement with other types of bone (see 2.3). For the forming bone, a broad WAXS peak at $q=19.4 \mathrm{~nm}^{-1}$ indicates the presence of an amorphous phase, where the short range ordering is reflected by a preferred distance of $3.23 \AA$ [53]. This spacing is not consistent with any known crystalline calcium phosphate mineral. A comparison of the SAXS signals with the form factor of spheres revealed that particles with a radius of about $6 \mathrm{~nm}$ are found in the forming bone (figure 5d) [53]. Note that the minima of the oscillations allow for an estimation of the radius, even though it was not possible to fit the entire scattering curve. Deviations between the calculated scattering function for the sphere model and the experimental data arise from relatively high background contributions caused by the surrounding tissue of the fin. In order to characterize the sample in a state which is as close as possible to the native one, the fish fin was used directly without separating the bony rays from the surrounding tissue. Giving access to the possibility of characterizing freshly dissected fins, the spatially resolved SAXS/WAXS studies were able to demonstrate that amorphous nanospheres act as a precursor during the formation of bone [53].

Similar concepts as described here for the zebrafish example could in the future help to also study possible precursor phases in mammalian bone. Moreover, the WAXS analysis can also be done in a quantitative manner allowing for mapping the local amount of HA and of amorphous calcium carbonate and phosphate minerals [56]. 
(a)

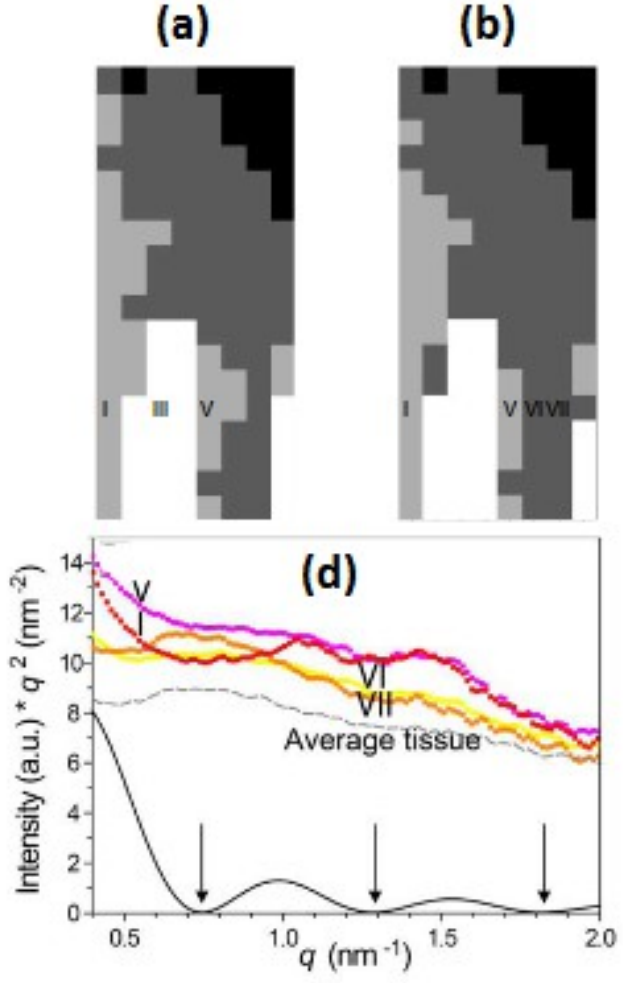

(c)

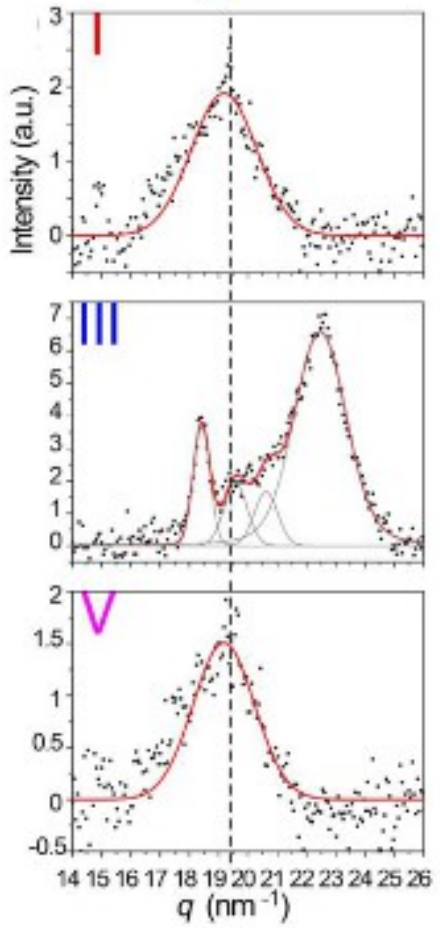

Figure 5: Scanning SAXS/WAXS of bony rays in zebrafish fins acquired with a $10 \mu \mathrm{m}$ beam. (a) Map obtained from WAXS analysis (white: bone apatite diffraction, light grey: mineral phase producing amorphous scatter, dark gray: organic tissue scattering, black: no signal, pixel size: $10 \mu \mathrm{m} \times 10 \mu \mathrm{m}$ ). (b) Map obtained from SAXS analysis (white: typical bone platelet scattering, light grey: spherical particles producing oscillatory scatter, dark grey: organic tissue scattering, black: no signal, pixel size: $10 \mu \mathrm{m} \times 10 \mu \mathrm{m}$ ). (c) Examples for WAXS patterns for the positions indicated in (a), I and V, broad peak of amorphous phase, III, apatite diffraction. Please note that the relatively broad peak in III results from the overlap of three peaks ((211), (112), (300)) of the known crystalline structure of HA. (d) Examples of SAXS patterns for the forming bone (I and V) as compared to the scattering of the surrounding organic tissue (VI, VII and averaged tissue). The minima due to the oscillations in I and V coincide with minima in the calculated SAXS of monodisperse spheres with a radius of $6 \mathrm{~nm}$ (lowermost curve, arrows). This figure was adapted from ref. [Mahamid et al., 2010] with permission from PNAS.

\subsection{Understanding specific bone functions by investigating the nanostructure in combination with other methods}

\subsubsection{Multi-scale and multi-physics approach}

From the structural point of view, several distinct levels of hierarchy can be observed in bone [57]. To answer bone-related research questions from both, an applied (e.g. medical or biological) as well as a basic research (e.g. physics, mechanics) viewpoint, usually different hierarchical levels (see section 1.3 in this chapter) have to be considered simultaneously during measurements. In this section, a combination of mechanical testing with X-ray scattering is described to illustrate how such combinations of methods with X-ray scattering can extend the accessible information about structurefunction relations in biological materials.

\subsubsection{Combining $x$-ray scattering and mechanical testing}

X-ray scattering can very well be combined with micromechanical testing, e.g. to measure the collagen fibril strain and correlate it simultaneously with the mineral particle strain as well as with the macroscopic tissue strain and stress in bone [58, 59]. Material level testing requires both a homogenous material and micron-sized samples. Homogenous bone material satisfying these conditions does not occur in every species, since most bone structures are rather diverse and complex 
[60]. Therefore, a specific model tissue like bovine fibrolamellar bone tissue has to be used. The percent change in the period D (see figure 3) during tensile testing, relative to the unstressed value, provides a measure of the fibril strain $\varepsilon_{\mathrm{F}}$ in bone (figure 6a-c) $[58,59]$. The (002) lattice spacing and its shift due to stretching the sample can be used to obtain the mineral strain parallel to the collagen fibril direction. The strain in the tissue is applied by a macroscopic tensile tester and measured by means of non-contact strain measurement by video extensometry. The maintenance of bone samples needs to be in a close to native state in phosphate buffered saline (PBS) to obtain reproducible results in quasi static loading experiments.

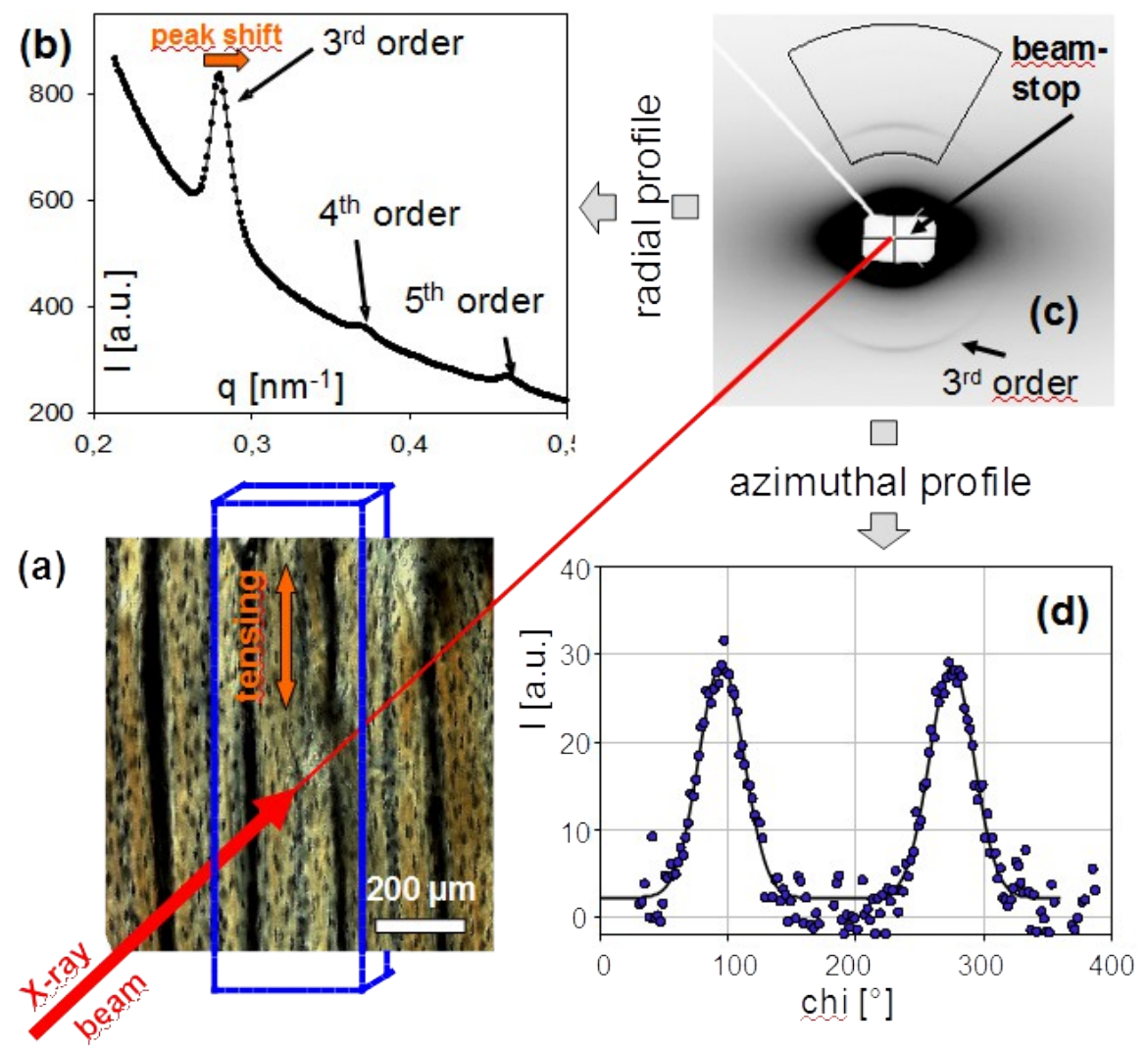

Figure 6: (a) polarized light microscopy image of a fibrolamellar bone sheet and schematic of specimen, (b) azimuthal intensity-chi plot of 3rd order collagen, (c) diffuse scattering and 3rd order collagen reflection, (d) radial integration (integration area shown in (c)).

The difficulty of measuring a true strain in biological samples is well known [61], and video extensometry techniques result in a significant improvement of the accuracy of the measured results compared with taking the strain from the nominal motor displacement. Combining this mechanical testing with scattering and diffraction presents additional technical issues, like air scattering and the absorption of X-rays in water. Therefore, such testing devices need to be built with minimal thickness in the beam path direction to minimize air scattering and absorption of the SAXS or WAXD signal in air (figure 7). Additionally, tubes covered with foil protrude into the fluid chamber to minimize the path (and consequently X-ray absorption and signal attenuation) of X-rays in water (figure 7c). Due to other installations and devices in a synchrotron hutch (like X-ray optics and vacuum tubes) the space is generally limited and the camera cannot look edge-on to the device. The overall design allows combining the use of a tensile tester and camera to fit in the given little user-area in a synchrotron user-hutch. The presented device (figure 7) is designed for in situ tensile testing on fibrolamellar bone but can easily be adapted to other bone tissues and different biomaterials which can be tested in situ in a wet environment. By using in situ tensile testing with synchrotron X-ray scattering on bone samples it could be shown that tissues, fibrils and mineral particles take up successively lower levels of strain $[58,59]$. In this work the tissue strain was measured by video extensometry, fibrillar strain by SAXS analysis and mineral strain by WAXS analysis concurrently during tensile loading of single fibrolamellar bone packets. 


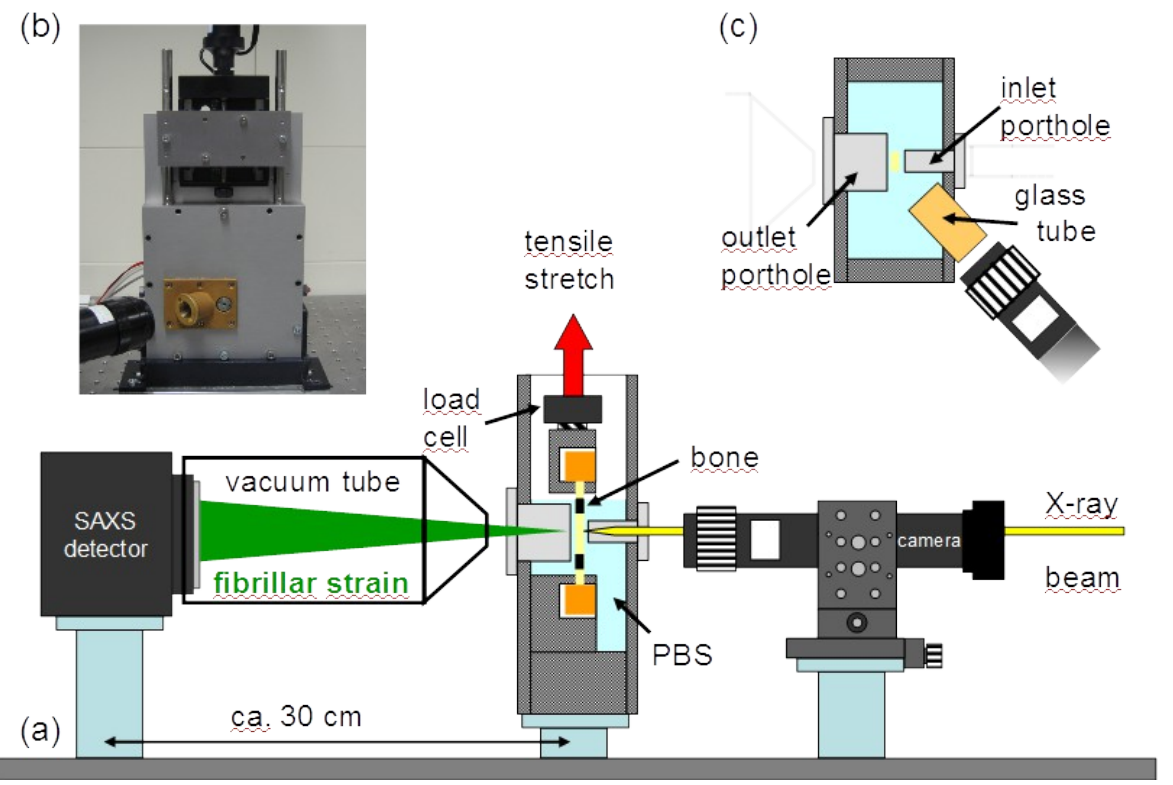

Figure 7: Schematic of the in-situ tensile testing setup (a) The tensile test is done in a fluid cell, filled with phosphate buffered saline (PBS) to immerse the sample throughout the test. Tensile strain is applied by moving the DC - encoder motor upward, while the load cell measures the force variation. The 2D area detector measures the small angle scattering pattern. (b) image of the tensile tester. (c) schematic of fluid chamber from top-view.

\subsection{Revealing the nanoscale properties of bone tissues and organs: scanning SAXS/WAXS imaging}

\subsubsection{Probing hierarchy by scanning}

In it's classical use, the main difference between SAXS/WAXS analysis and other high-resolution techniques, such as (HR)TEM, is that it provides a global statistical view of the system rather than a detailed local picture. The structural information obtained relates to the ensemble of mineral nanoparticles in the bone matrix within the sample volume illuminated by the X-ray beam. However, one can greatly improve this description if using small $\mathrm{X}$-ray beams relative to the sample size. In particular, if the beam diameter is in the same size range of the microstructural features, a local analysis can be performed. Furthermore, by using appropriate scanning devices it becomes possible to acquire a series of 2D SAXS/WAXS patterns as a function of the sample position. This category of experiments falls in the broad class of scanning small- and wide-angle $X$-ray scattering (sSAXS/sWAXS) measurements.

\subsubsection{Instrumental aspects of scanning experiments using X-ray microbeams}

Set aside the sample scattering power (see section 2.1), the potential for mapping depends essentially on three instrumental parameters: the beam size at the sample position, the X-ray flux and the detector speed. Due to the fact that the refraction index in materials is very close to $n=1$, it is extremely difficult, in practice, to make X-ray lenses in the classical sense, as is the case for light or even electron microscopy. Nevertheless, it is still possible to use devices to shape or focus X-ray beams, which are generally grouped under the generic term of X-ray optics [62]. The simplest elements are combinations of slits, pinholes and/or collimators which absorb the outermost part of the incident beam and which geometry ultimately defines the beam size and divergence. Other examples include mirrors, capillaries and waveguides which can be used in grazing incidence condition to force the incident Xrays to converge to the sample position by reflection. In the last decade, important progress has also been made using the very small difference in refraction index to the value of 1 . This is the case of parabolic lenses generally used in series. More recently, new optics for X-rays have been developed using phase interference such as zone plates or Fresnel and Bragg-Fresnel lenses, using well known principles in optics. Those technological developments constitute the basis for scanning X-ray scattering with microbeams $[5,63]$. 
The X-ray flux has increased by orders of magnitude with the advent of high brilliance $3^{\text {rd }}$ generation synchrotron sources, and is still constantly improving because of better insertion devices such as invacuum undulators, pulsed sources etc. However, for focused X-ray beams, the flux can be considerably reduced depending on the optics acceptance. This is easily understood in the case of pinholes which simply allow a small part of the incident beam to go through, absorbing the rest. Therefore, the choice of the optics, which ultimately defines the beam size, strongly depends on the source properties. In general, smaller beam sizes are obtained at the cost of X-ray flux, which partly explains why laboratory instruments are typically limited to beams $>50 \mu \mathrm{m}$ in diameter for scattering experiments. This is already sufficient to distinguish trabecular bone from the cortical shell (figure 1). Microfocus beamlines for SAXS/WAXS studies at second generation sources, such as BESSY, SOLEIL, SLS, typically provide beam sizes in the order of $10-20 \mu \mathrm{m}$, allowing to spatially resolve osteons in cortical bone. So far, beams $<1 \mu \mathrm{m}$ are only available at $3^{\text {rd }}$ generation synchrotrons such as the ESRF and tests have already demonstrated that focusing as small as $50 \mathrm{~nm}$ could be achieved with reasonable flux $\left(>10^{8} \mathrm{ph} \cdot \mathrm{s}^{-1}\right)$. These length scales could clearly be sufficient to obtain very fine maps of osteons, canalicules and in the best case, could be used to map single microfibrils ( $\varnothing \sim 100 \mathrm{~nm}$ ). An illustration of the characteristic beam size needed to spatially resolve the structural features of interest is shown in figure $8 \mathrm{a}, 8 \mathrm{~b}$ (top) and figure $8 \mathrm{c}$ (left). Images in figure8a, $8 \mathrm{~b}$ (top) were obtained at the nanofocus beamline of the ESRF, ID13 and at the cSAXS beamline of the SLS.
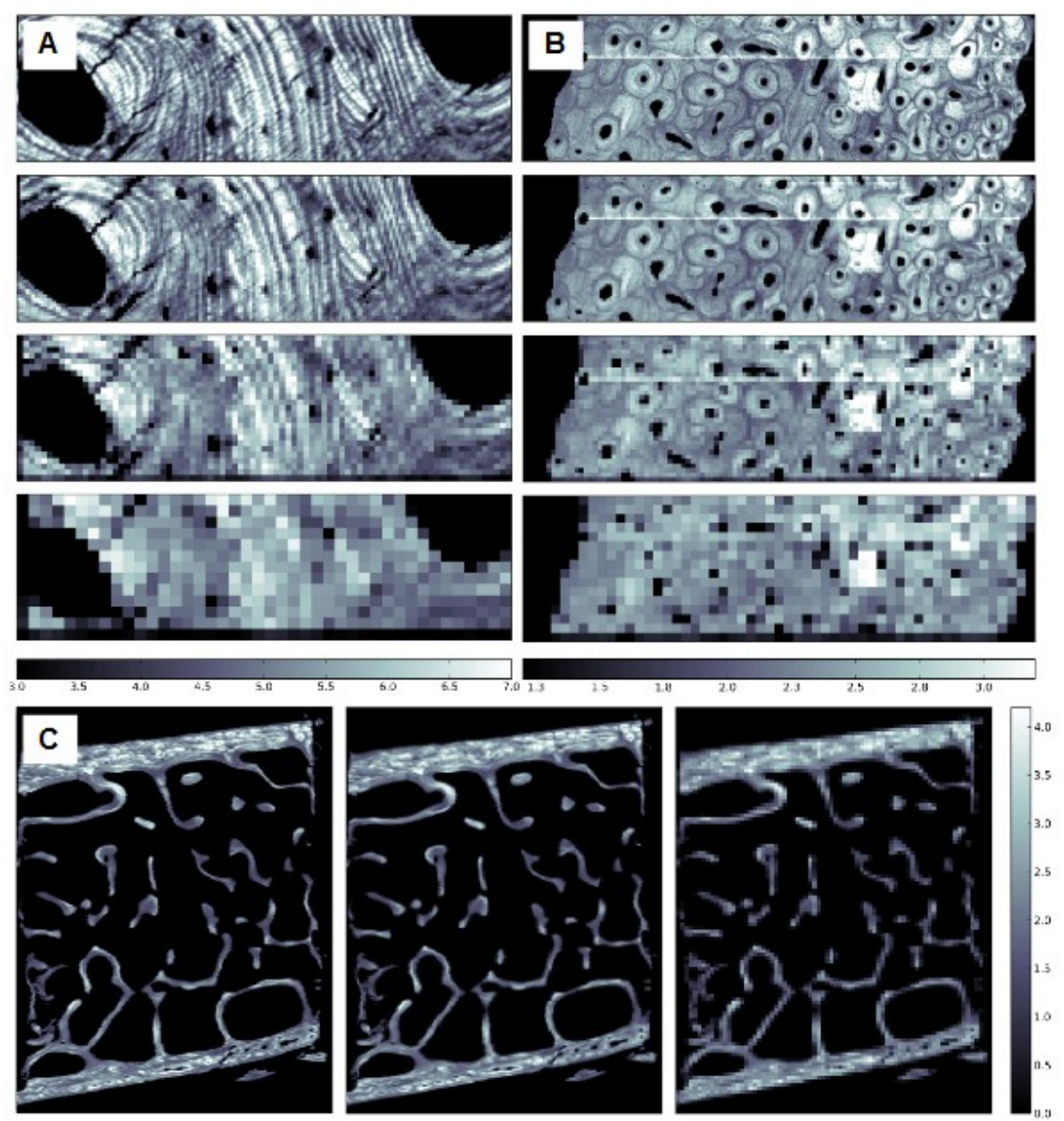

Figure 8: q-sSAXS images of the integrated SAXS intensity J (a.u.; see eq.xx) showing the impact of beam size on spatial resolution and field of view: a) region between two osteons in the cortical region of a transverse thin transverse section $(40 \mu \mathrm{m})$ of a human femoral diaphysis $\left(\varnothing_{\mathrm{H}, \mathrm{V}}=1 \mu \mathrm{m} ; \mathrm{s}_{\mathrm{H}, \mathrm{V}}=1 \mu \mathrm{m} ; \mathrm{A}_{\mathrm{H}}=350 \mu \mathrm{m} ; \mathrm{A}_{\mathrm{V}}=100 \mu \mathrm{m}\right)$ in the native format (top) and binned (below) by a factor 2, 4 and 8 from top to bottom [48]; b) full map across a the cortical section of a similar sample as a) obtained from a different individual $\left(\varnothing_{\mathrm{H}, \mathrm{V}}=5 \mu \mathrm{m} ; \mathrm{s}_{\mathrm{H}, \mathrm{V}}=8 \mu \mathrm{m} ; \mathrm{A}_{\mathrm{H}}=4 \mathrm{~mm}\right.$; $\left.A_{V}=1 \mathrm{~mm}\right)$ with the same binning convention [48]; c) thin section $(80 \mu \mathrm{m})$ of an iliac crest biospy of a sheep $\left(\varnothing_{\mathrm{H}}=20 \mu \mathrm{m} ; \varnothing_{\mathrm{V}}=12 \mu \mathrm{m} ; \mathrm{s}_{\mathrm{H}}=40 \mu \mathrm{m} ; \mathrm{s}_{\mathrm{H}, \mathrm{V}}=20 \mu \mathrm{m} ; \mathrm{A}_{\mathrm{H}}=10 \mathrm{~mm} ; \mathrm{A}_{\mathrm{V}}=6 \mathrm{~mm}\right)$ in the native format (left) and binned (right) by a factor 2, 4 [49]. 
The choice of X-ray optics also calls for additional comments. First the beam size isn't the only parameter to be considered since X-ray scattering experiments, particularly SAXS, require a low divergence $(<1 \mathrm{mrad})$. A detailed explanation for this last point is beyond the scope of this chapter and is well described in other textbooks (e.g. [64,65]). It suffices, here, to state that certain types of optics should not be considered for scanning SAXS experiments because of a too high divergence. Secondly, the beam size is generally defined by it's vertical and horizontal full width at half-maximum (FWHM) and considered to have a gaussian shape in first approximation. But it is important to bear in mind, when performing an experiment that the beam shape strongly depends on the optical system and can be quite irregular in 3D. In particular, it is important to consider that the tails of the beam can extend relatively far, implying that a measured point might have received a non-negligible dose from the previous scanned point. This is important if the sample is sensitive to radiation damage.

\subsubsection{Qualitative versus quantitative sSAXS/WAXS imaging}

The acquisition procedure in scanning mode is very similar to other well known microspectroscopy methods such as micro-Raman, -infrared or -fluorescence imaging. Such hyperspectral techniques provide 3D data sets with two dimensions for the scan coordinates and a third for the measured spectra. The imaging process itself relies on the reduction of the 1D spectra to scalar values representing a structural or chemical feature, e.g. the band position or intensity relative to the presence or the quantity of a given chemical element in the sample. In the case of scanning X-ray scattering (sSAXS/WAXS), the problem is slightly more complex since a 2D image is acquired at each scan coordinate, thus resulting in a $4 \mathrm{D}$ data set. As a result, there are two levels of spatial resolutions: in reciprocal space, where it is mostly defined by the sample-to-detector geometry and in real space, defined by the beam size and scan steps. Therefore, sSAXS/WAXS imaging provides two levels of structural details: nanoscale (atomic and molecular) from the SAXS/WAXS patterns and mesomacroscale from the scanning procedure. Hence, the main strength of this technique resides in the fact that one can obtain images of nanoscale parameters with fields of view extending by several orders of magnitude those generally accessible with other high-resolution imaging methods.

In the literature, the terms mapping or imaging are used indifferently to define the result of the sSAXS/WAXS process. In our view, there is a subtle distinction between the two, which essentially lies in the ability to analyze the 2D scattering patterns in an automated way and in real time. Online imaging therefore implies that the SAXS/WAXS data analysis/reduction must be performed at a speed close to the acquisition frame rate. In many cases, a preliminary step needs to be taken, e.g. in order to define initial analysis parameters. This leads to so-called quasi-online imaging. Therefore, the key to (online) imaging, resides in the use and development of specific software for the analysis of very large SAXS/WAXS data sets. As an example, the left image of figure 8c was obtained by analysis of 140896 2D frames $(296(\mathrm{H}) \times 476(\mathrm{~V}))$ of $2.44 \mathrm{MB}$, amounting to a full data set of $\sim 345 \mathrm{~GB}$. As will be discussed in the next section, a realistic figure for the analysis time for one frame should be $<100 \mathrm{~ms}$. Even with optimized calculation algorithms, it should be clear that the data analysis must be kept as simple as possible and clearly excludes heavy computing operations such as complex modeling, typically used for the SAXS analysis of, say, proteins in solutions.

A simple and elegant approach was proposed by Bunk et al., making use of integrated values of the intensity contained in a ROI $(\Delta q, \Delta \chi)$ of the SAXS/WAXS data [66]. This simple procedure fits well with the above criteria and was termed scanning-SAXS imaging (sSAXSI). The contrast in the images obtained in this way therefore originates from structural differences at the nanoscale in heterogeneous sample. However, the information is mostly qualitative, since it is not always clear exactly what is the structural source of contrast.

In contrast to the previous method, one can also make extensive use of the theory of SAXS/WAXS analysis to calculate structural parameters of interest. As discussed in section 2, in the case of bone, as in most mineralized biological tissues, the problem reduces to the well known case of a two-phased media (organic and mineral) with sharp boundaries. Hence, the calculation of so-called integral parameters, which is computationally relatively straightforward, provides a direct access to a range of meaningful structural parameters such as $T$ (equation 5) [41]. Hence, this method is highly 
quantitative and will be referred to as quantitative scanning-SAXS imaging (q-SSAXSI). By analogy with other micro-imaging techniques, we thus use the term imaging resolution to define the pixel size of the images, which quantifies the value of the structural parameter derived from the analysis, and field of view as the extent of the image, i.e. of the scan.

\subsubsection{Image resolution versus field of view}

The first experimental parameter to determine when planning a q-SSAXSI experiment is the maximum beam size to be used for a given experiment in order to spatially resolve the structural features of interest in the final q-sSAXS (or WAXS) images. Each image shown in figure 8 is also displayed binned by a factor 2, 4 and 8 . To a good approximation, those correspond to images that would have been obtained with beam sizes enlarged by the same factor. It can clearly be seen that the dark and bright lamellae in figure $8 \mathrm{a}$, which relate to differences in microfibril orientation [41], can only be observed for beam diameters of $<2-3 \mu \mathrm{m}$. Similarly, the osteons and bone packets in figure $8 \mathrm{~b}$ can only be distinguished clearly with beams $\leq 10 \mu \mathrm{m}$, while cortical and trabecular bone are better observed with beams $\leq 50 \mu \mathrm{m}$.

Then, the extent of the map needs to be defined, i.e. how many 2D SAXS/WAXS frames are to be collected in horizontal and vertical directions and what distance should be chosen between two consecutive rows and columns. Given a set of experimental restrictions, including the sample scattering power, the maximum number of data that can be collected ultimately depends on the detector speed. In the last decades, priority has been given, in synchrotron, developments to improvements of the brilliance of the source and the X-ray optics. Only recently was the relative importance of 2D detectors realized, leading to considerable developments. From charge-coupled devices (CCDs) to single photon counting detectors, the characteristic figures in sensitivity, dynamic range and read-out time have improved by orders of magnitude.

In practice, the resolution in direct space, i.e. the size of a pixel in horizontal or vertical directions $p_{H, V}$ of the reconstructed image, is defined by the convolution of the beam dimensions $\emptyset_{H, V}$ and the scan steps $s_{H, V}: p_{H, V}=\varnothing_{H, V} \otimes s_{H, V}$. The field of view (FOV), corresponding to the scanned sample area $A$ is, then, defined by: $A=n_{H} \cdot p_{H} \times n_{V} \cdot p_{V}$, where $n_{H, V}$ is the number of scan steps. If $s_{H, V}=\varnothing_{H, V}$, the sample is said to be scanned with full resolution and $p_{H, V}=\varnothing_{H, V}$. Otherwise if $s_{H, V}>\varnothing_{H, V}$ or $s_{H, V}<\varnothing_{H, V}$ one speaks of undersampling or oversampling respectively. Of the latter two cases, the first is often chosen to gain time in the overall scan or extend the field of view at the cost of a reduced spatial resolution, while the second is more rarely used to increase the spatial resolution by deconvolution procedures.

Thus, the frequency of acquisition $f$ can be defined as:

$$
f=A /\left(p_{H} \cdot p_{V} \cdot t\right)=n_{H} \cdot n_{V} / t \quad \text { (equation 9) }
$$

where $t$ is the acquisition time for each frame.

In the case of a full resolution scan, and assuming identical dimensions in horizontal and vertical directions for the beam size $\left(\varnothing_{H}=\varnothing_{V}\right)$ and the extent of the scan $\left(n_{H}=n_{V}\right)$, eq. 9 reduces to:

$$
f=A /\left(\phi^{2} . t\right)=n^{2} / t \quad \text { (equation 10) }
$$

Hence, in order to scan a region of $1 \mathrm{~cm}^{2}$ sample with a beam of $10 \times 10 \mu \mathrm{m}^{2}$ (case of figure $8 \mathrm{c}$ ) in $2 \mathrm{~h}$, one must acquire data with a frequency of $f=138 \mathrm{~Hz}$. It is worthwhile noting that $\mathrm{A}$ ) a $1 \mathrm{~cm}^{2}$ region typically corresponds to the the size of a transverse section of an illiac crest biopsy or of the anterior/posterior region of human cortical bone from the femoral diaphysis; B) $10 \mu \mathrm{m}$ was chosen as the minimum resolution to resolve secondary osteons which are typically 50-100 $\mu \mathrm{m}$ in diameter; C) 2 $\mathrm{H}$ should be a reasonable scan time for 1 sample in the framework of a biomedical study on a large set of biopsies (cohort). Conversely, given a maximum acquisition frame rate of $30 \mathrm{~Hz}$ and keeping all other parameters identical, the beam size can be calculated from eq. 10 as $\varnothing_{H, V}=22 \mu \mathrm{m}$, which still allows resolving the largest osteons. 
Ideally, in sSAXS/WAXS experiments, the goal is to achieve the largest possible field of view with the highest possible spatial resolution. However, the above considerations clearly show that, as with any other scanning imaging technique, there is a tradeoff between the two. It is therefore essential not to overestimate the spatial resolution needed. The figures indicated in table 1 provide rough guidelines to decide which beam size should be used for an experiment.

\begin{tabular}{|c|c|c|c|c|}
\hline \multirow{2}{*}{$\mathrm{f}(\mathrm{Hz})$} & \multicolumn{4}{|c|}{$\varnothing(\mu \mathrm{m})$} \\
\cline { 2 - 5 } & 0.1 & 1 & 10 & 50 \\
\hline 1 & $6(36)$ & $60(3600)$ & $600(3.6 \mathrm{E} 5)$ & $3000(9 \mathrm{E} 6)$ \\
\hline 10 & $19(360)$ & $190(3.6 \mathrm{E} 4)$ & $1897(3.6 \mathrm{E} 6)$ & $9486(1.8 \mathrm{E} 8)$ \\
\hline 30 & $33(1080)$ & $329(1.08 \mathrm{E} 5)$ & $3286(1.08 \mathrm{E} 8)$ & $16432(2.7 \mathrm{E} 10)$ \\
\hline
\end{tabular}

Table 1: values of the accessible scan width, $n . \varnothing$ (field of view, $A)$ in $\mu \mathrm{m}\left(\mu \mathrm{m}^{2}\right)$ as a function of beam dimensions and acquisition frequency; the values were calculated using eq. 10 with $t=1 \mathrm{~h}$.

Finally, it should be emphasized that, ultimately, the optimization of the acquisition frequency depends on the signal-to-noise ratio which can become critical at low exposure times. The camera sensitivity then becomes a critical issue.

\subsubsection{Digital image processing of q-sSAXSI}

When confronted with a problem relating to the ultrastructure of bone, q-SSAXSI measurements should be envisaged as part of a more general study. The design of the experiments requires selecting the X-ray beam-size, scan step and extent of the scan depending on the available instrumental setups according to section 4.1 and table 1. The q-sSAXS images derived from the analysis reveal details of the nanoscale structure throughout the sample region scanned in the beam as shown in figure 9. Thus, once such images are obtained, further analysis needs to be performed to relate the nanoscale information to the histo-morphological features observed and gain a broader understanding of the sample properties. This is illustrated in the example shown in figure 9a showing images of $T$ (particle thickness in first approximation) of bovine bone sections submitted to a heat treatment at 100, 150, 170, 190, 210 and $250^{\circ} \mathrm{C}$ for 1 hour [67]. The q-SSAXSI experiments were performed at the cSAXS beamline at SLS using a monochromatic beam of $25(\mathrm{H}) \times 6(\mathrm{~V}) \mu \mathrm{m}$ in size (FWHM) at an energy of $18.58 \mathrm{keV}$. A scan step of $50(\mathrm{H}) \times 20(\mathrm{~V})$ was selected in order to map the whole cortical thickness $(\sim 11.5 \mathrm{~mm})$ within a $1.5 \mathrm{~mm}$ wide stripe. The images are displayed using the same color scale to highlight the local and global differences in the nanoparticle thicknesses.

The first observation is the presence of clear histological features in all images, including osteons, haversian canals and interstitial bone. This tends to show that, independently of any possible structural changes at the nanoscale, the microstructure is preserved at temperatures as high as $250{ }^{\circ} \mathrm{C}$. Secondly, there is an overall increase in image brightness indicating that, on average, the particle size increases. Furthermore, the contrast between bright and darker region seems to increase as a function of temperature. In order to quantify those changes, we followed, here, a method developed for the analysis of the bone mineral density distribution (BMDD) by Roschger et al. [68] making use of image histograms. This is common practice in digital image analysis methods and provides a statistical view of the image irrespectively of the sample morphology. Figure $9 \mathrm{~b}$ shows the corresponding histograms which take the form of Gaussian functions in first approximation. Clearly, the position of the maximum, $T_{M A X}$, indicating the mean particle thickness, shifts towards higher values as a function of temperature. Additionally, the width of the curves, $T_{F W H M}$, gets larger, pointing to a significant increase in the dimensional spread, which implies that the particle growth is not homogeneous throughout the sample. The results, summarized in figure $9 \mathrm{c}$, reveal an exponential trend for the two parameters. 
Such analysis should be viewed as a first step towards more sophisticated types of investigations such as image segmentation and classification. However, this study highlights the importance of q-sSAXSI for direct experimental multiscale analysis of bone, since the nanoscale parameters can be directly compared with the histo-morphological features of the images those obtained by other modalities.

Based on the previous example of artificially heated bone, a similar approach was adopted for the analysis of archaeological bone samples. The complexity related to this field of application is that there is generally limited information available to put the bone fragments discovered on the field in a general context. Additionally, in most cases, they are moderately to severely alter by complex physical, geochemical and biological processes. Hence, the interpretation of any micro-analysis performed on such samples is a tedious task due to the necessity to combine the results obtained with other modalities.
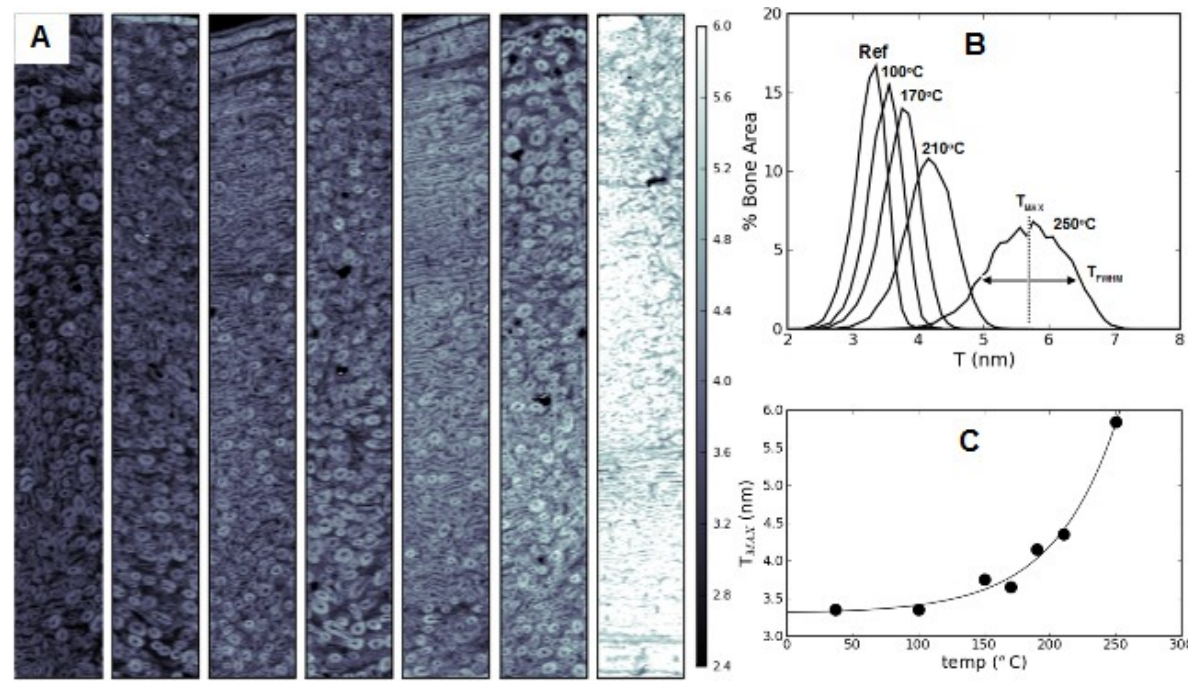

Figure 9: Schematic of the in-situ tensile testing setup (a) The tensile test is done in a fluid cell, filled with phosphate buffered saline (PBS) to immerse the sample throughout the test. Tensile strain is applied by moving the DC - encoder motor upward, while the load cell measures the force variation. The 2D area detector measures the small angle scattering pattern. (b) image of the tensile tester. (c) schematic of fluid chamber from top-view.

Figure 10 shows the histograms of q-sSAXS images acquired in similar conditions as the previous example on a series of archaeological samples obtained from the archaeological lake site of Chalain [69]. Since the histological details of the images cannot be put in perspective, the histograms provide the best source of comparison between the samples. Due to the differences in histogram shape and position, this study concluded that the nanoscale mineral properties could be used as structural markers to evaluate the state of conservation of the objects.

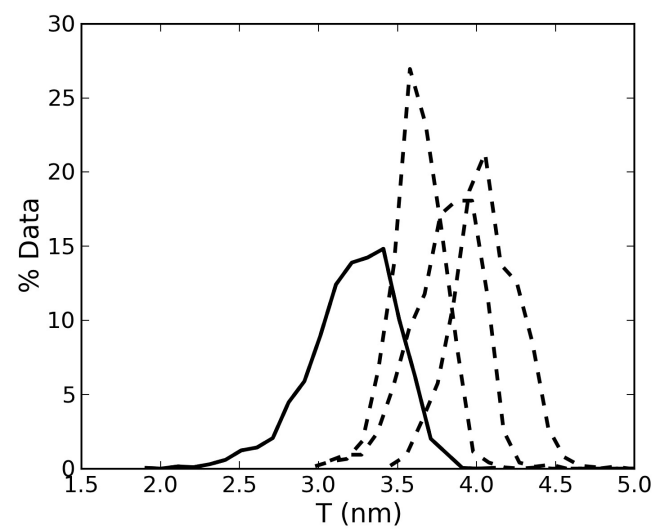

Figure 10: Schematic of the in-situ tensile testing setup (a) The tensile test is done in a fluid cell, filled with phosphate buffered saline (PBS) to immerse the sample throughout the test. Tensile strain is applied by moving the DC - encoder motor upward, while the load cell measures the force variation. The $2 \mathrm{D}$ area detector measures the small angle scattering pattern. (b) image of the tensile tester. (c) schematic of fluid chamber from top-view. 
The previous sets of examples illustrate one very important advantage of q-SSAXSI: the possibility to relate nanoscale parameters to higher levels of the hierarchy. It is no doubt that, in the future, more refined image analysis processes applied to q-SSAXSI will open new perspectives in fundamental and applied areas of research on bone.

\subsubsection{Scanning vs full-field SAXS imaging}

In the previous sub-sections, we have described how the scattering contrast could be used to image nanoscale structural parameters in bone. The fundamental mechanisms of the method lie in the systematic exploitation of the sample X-ray scattering. In scanning mode, this is done either in a qualitative way, by integrating a ROI in reciprocal space, or qualitatively, by analyzing the SAXS pattern with the available theoretical tools. However, it is worthwhile noting that other techniques exist that also make use of scattering as a source of contrast, but are full-field methods. In this case, the whole sample is illuminated by an X-ray source, similar to well-known radiography techniques. The main difference between the scanning and full-field techniques lies in the way the scattering signal is exploited. It is beyond the scope of this chapter to provide a detailed description of available full-field methods, but it is important to note that all those techniques rely on the integration of the scattering signal of poorly defined regions of reciprocal space. Dark-field X-ray imaging, for instance, produces images where, in theory, all scattering effects are averaged, independent of their structural origins [70]. X-ray interferometry attempts to alleviate this limitation by limiting the $q$-range (and often $\chi$ range) of the measurement [71], but a reconstruction of the reciprocal-space information is a very difficult challenge still untackled to this day. Although very informative, such methods therefore remain essentially qualitative in nature. Due to the instrumental improvements described in section 4.1, scanning X-ray scattering imaging methods thus remain very competitive for the study of the ultrastructure of bone.

\section{References}

1. Lakes, R., Materials with Structural Hierarchy. Nature, 1993. 361(6412): p. 511-515.

2. Fratzl, P., et al., Structure and mechanical quality of the collagen-mineral nano-composite in bone. Journal of Materials Chemistry, 2004. 14(14): p. 2115-2123.

3. Weiner, S., W. Traub, and H.D. Wagner, Lamellar bone: Structure-function relations. Journal of Structural Biology, 1999. 126(3): p. 241-255.

4. Nakano, T., et al., Unique alignment and texture of biological apatite crystallites in typical calcified tissues analyzed by microbeam X-ray diffractometer system. Bone, 2002. 31(4): p. 479-487.

5. Paris, O., et al., Analysis of the hierarchical structure of biological tissues by scanning X-ray scattering using a microbeam. Cellular and Molecular Biology, 2000. 46(5): p. 993-1004.

6. Wenk, H.R. and F. Heidelbach, Crystal alignment of carbonated apatite in bone and calcified tendon: Results from quantitative texture analysis. Bone, 1999. 24(4): p. 361-369.

7. Orgel, J.P.R.O., et al., The in situ supermolecular structure of type I collagen. Structure, 2001. 9(11): p. 1061-1069.

8. Wess, T.J., Collagen fibril form and function. Fibrous Proteins: Coiled-Coils, Collagen and Elastomers, 2005. 70: p. 341.

9. Weiner, S. and H.D. Wagner, The material bone: Structure mechanical function relations. Annual Review of Materials Science, 1998. 28: p. 271-298.

10. Rubin, M.A., et al., TEM analysis of the nanostructure of normal and osteoporotic human trabecular bone. Bone, 2003. 33(3): p. 270-282.

11. Landis, W.J., et al., Structural relations between collagen and mineral in bone as determined by high voltage electron microscopic tomography. Microscopy Research and Technique, 1996. 33(2): p. 192-202.

12. Enlow, D.H. and S.O. Brown, A comparative histological study of fossil and recent bone tissues. Texas Journal of Science, 1956. 8: p. 405-443.

13. Weiner, S. and L. Addadi, Design strategies in mineralized biological materials. Journal of Materials Chemistry, 1997. 7(5): p. 689-702.

14. Stribeck, N., X-ray scattering of soft matter. 2007, Berlin ; New York: Springer. xx, 238 p. 
15. Sasaki, N., et al., Time-resolved X-ray diffraction from tendon collagen during creep using synchrotron radiation. Journal of Biomechanics, 1999. 32(3): p. 285-292.

16. Petruska, J.A. and A.J. Hodge, Subunit Model for Tropocollagen Macromolecule. Proceedings of the National Academy of Sciences of the United States of America, 1964. 51(5): p. 871.

17. Bragg, W.L., The analysis of crystals with an $x$-ray spectrometer. Zeitschrift Fur Anorganische Chemie, 1914. 90(2/3): p. 246-269.

18. Holzwarth, U. and N. Gibson, The Scherrer equation versus the 'Debye-Scherrer equation'. Nature Nanotechnology, 2011. 6(9): p. 534-534.

19. Langford, J.I. and A.J.C. Wilson, Scherrer after 60 Years - Survey and Some New Results in Determination of Crystallite Size. Journal of Applied Crystallography, 1978. 11(Apr): p. 102-113.

20. Danilchenko, S.N., et al., Determination of the bone mineral crystallite size and lattice strain from diffraction line broadening. Crystal Research and Technology, 2002. 37(11): p. 1234-1240.

21. Schäfer, W., Textur vielkristalliner Materie - Anwendungen in Werkstoff- und Geowissenschaften. Report Forschungszentrum Jülich Streumethoden zur Untersuchung kondensierter Materie., 1996: p. pp.C4.1-C4.16.

22. Randle, V. and O. Engler, Introduction to texture analysis : macrotexture, microtexture and orientation mapping. 2000, Amsterdam, The Netherlands: Gordon \& Breach. 388.

23. Ascenzi, A., et al., X-ray diffraction on cyclically loaded osteons. Calcified Tissue International, 1998. 62(3): p. 266273.

24. Heidelbach, F., C. Riekel, and H.R. Wenk, Quantitative texture analysis of small domains with synchrotron radiation X-rays. Journal of Applied Crystallography, 1999. 32: p. 841-849.

25. Sasaki, N. and Y. Sudoh, X-ray pole figure analysis of apatite crystals and collagen molecules in bone. Calcified Tissue International, 1997. 60(4): p. 361-367.

26. Bacon, G.E., P.J. Bacon, and R.K. Griffiths, Orientation of Apatite Crystals in Bone. Journal of Applied Crystallography, 1979. 12(Feb): p. 99-103.

27. Jaschouz, D., et al., Pole figure analysis of mineral nanoparticle orientation in individual trabecula of human vertebral bone. Journal of Applied Crystallography, 2003. 36: p. 494-498.

28. Wagermaier, W., et al., Spiral twisting of fiber orientation inside bone lamellae. Biointerphases, 2006. 1(1): p. 1-5.

29. Wagermaier, W., et al., Scanning texture analysis of lamellar bone using microbeam synchrotron X-ray radiation. Journal of Applied Crystallography, 2007. 40: p. 115-120.

30. Glimcher, M. and S. Krane, Treatise on Collagen, B. Gould, Editor. 1968, Academic Press: New York. p. 67-251.

31. Hodge, A.J. and J.A. Petruska, Aspect of Protein Structure, G. Ramachandran, Editor. 1963, Academic Press: New York. p. 289-300.

32. Robinson, R.A., An Electron-Microscopic Study of the Crystalline Inorganic Component of Bone and Its Relationship to the Organic Matrix. Journal of Bone and Joint Surgery-American Volume, 1952. 34-A(2): p. 389-\&.

33. Weiner, S. and W. Traub, Bone-Structure - from Angstroms to Microns. Faseb Journal, 1992. 6(3): p. 879-885.

34. Ziv, V. and S. Weiner, Bone Crystal Sizes - a Comparison of Transmission Electron-Microscopic and X-RayDiffraction Line-Width Broadening Techniques. Connective Tissue Research, 1994. 30(3): p. 165-175.

35. Kratky, O., G. Porod, and L. Kahovec, *EINIGE NEUERUNGEN IN DER TECHNIK UND AUSWERTUNG VON RONTGEN-KLEINWINKELMESSUNGEN. Zeitschrift Fur Elektrochemie, 1951. 55(1): p. 53-59.

36. Porod, G., DIE RONTGENKLEINWINKELSTREUUNG VON DICHTGEPACKTEN KOLLOIDEN SYSTEMEN . 1. Kolloid-Zeitschrift and Zeitschrift Fur Polymere, 1951. 124(2): p. 83-114.

37. Porod, G., DIE RONTGENKLEINWINKELSTREUUNG VON DICHTGEPAKTEN KOLLOIDEN SYSTEMEN .2. Kolloid-Zeitschrift and Zeitschrift Fur Polymere, 1952. 125(1): p. 51-57.

38. Fratzl, P., et al., NUCLEATION AND GROWTH OF MINERAL CRYSTALS IN BONE STUDIED BY SMALLANGLE X-RAY-SCATTERING. Calcified Tissue International, 1991. 48(6): p. 407-413.

39. Fratzl, P., et al., MINERAL CRYSTALS IN CALCIFIED TISSUES - A COMPARATIVE-STUDY BY SAXS. Journal of Bone and Mineral Research, 1992. 7(3): p. 329-334.

40. Rinnerthaler, S., et al., Scanning small angle X-ray scattering analysis of human bone sections. Calcified Tissue International, 1999. 64(5): p. 422-429.

41. Gourrier, A., et al., Scanning X-ray imaging with small-angle scattering contrast. Journal of Applied Crystallography, 2007. 40: p. S78-S82. 
42. Zizak, I., et al., Characteristics of mineral particles in the human bone/cartilage interface. Journal of Structural Biology, 2003. 141(3): p. 208-217.

43. Fratzl, P., S. Schreiber, and K. Klaushofer, Bone mineralization as studied by small-angle $x$-ray scattering. Connective Tissue Research, 1996. 35(1-4): p. 9-16.

44. Fratzl, P., et al., Diffracting stacks of cards - some thoughts about small-angle scattering from bone. Progress in Colloid and Polymer Science, 2005. 130: p. 273-291.

45. Gourrier, A., et al., Scanning small-angle X-ray scattering analysis of the size and organization of the mineral nanoparticles in fluorotic bone using a stack of cards model. Journal of Applied Crystallography, 2010. 43: p. 13851392.

46. Bunger, M.H., et al., Strontium and Bone Nanostructure in Normal and Ovariectomized Rats Investigated by Scanning Small-Angle X-Ray Scattering. Calcified Tissue International, 2010. 86(4): p. 294-306.

47. Burger, C., et al., Lateral packing of mineral crystals in bone collagen fibrils. Biophysical Journal, 2008. 95(4): p. 1985-1992.

48. Lange, C., et al., Fetal and postnatal mouse bone tissue contains more calcium than is present in hydroxyapatite. Journal of Structural Biology, 2011. 176(2): p. 159-167.

49. Cipitria, A., et al., Porous scaffold architecture guides tissue formation. Journal of Bone and Mineral Research, 2012. 27(6): p. 1275-1288.

50. Liu, Y.F., et al., Mineral crystal alignment in mineralized fracture callus determined by 3D small-angle X-ray scattering. Xiv International Conference on Small-Angle Scattering (Sas09), 2010. 247.

51. Seidel, R., et al., Synchrotron 3D SAXS analysis of bone nanostructure. Bioinspired, Biomimetic and Nanobiomaterials, 2011. 1(BBN2): p. 123-132.

52. Addadi, L., S. Raz, and S. Weiner, Taking advantage of disorder: Amorphous calcium carbonate and its roles in biomineralization. Advanced Materials, 2003. 15(12): p. 959-970.

53. Mahamid, J., et al., Mapping amorphous calcium phosphate transformation into crystalline mineral from the cell to the bone in zebrafish fin rays. Proceedings of the National Academy of Sciences of the United States of America, 2010. 107(14): p. 6316-6321.

54. Pokroy, B., A.N. Fitch, and E. Zolotoyabko, The microstructure of biogenic calcite: A view by high-resolution synchrotron powder diffraction. Advanced Materials, 2006. 18(18): p. 2363-2368.

55. Pedersen, J.S., Analysis of small-angle scattering data from colloids and polymer solutions: modeling and least-squares fitting. Advances in Colloid and Interface Science, 1997. 70: p. 171-210.

56. Bentov, S., et al., Enamel-like apatite crown covering amorphous mineral in a crayfish mandible. Nature Communications, 2012. 3: p. 839.

57. Rho, J.Y., L. Kuhn-Spearing, and P. Zioupos, Mechanical properties and the hierarchical structure of bone. Medical Engineering \& Physics, 1998. 20(2): p. 92-102.

58. Gupta, H.S., et al., Cooperative deformation of mineral and collagen in bone at the nanoscale. PNAS, 2006. 103(47): p. 17741-6.

59. Gupta, H.S., et al., Nanoscale Deformation Mechanisms in Bone. Nano Letters, 2005. 5(10): p. 2108-2111.

60. Currey, J.D., The many adaptations of bone. Journal of Biomechanics, 2003. 36(10): p. 1487-1495.

61. Burgert, I., et al., Microtensile testing of wood fibers combined with video extensometry for efficient strain detection. Holzforschung, 2003. 57(6): p. 661-664.

62. Erko, A.I., Modern developments in X-ray and neutron optics. 2008, Berlin ; New York: Springer. xxiii, 533 p.

63. Riekel, C., New avenues in $x$-ray microbeam experiments. Reports on Progress in Physics, 2000. 63(3): p. $233-262$.

64. Glatter, O. and O. Kratky, Small angle X-ray scattering. 1982, London: Academic. x, 515 p.

65. Guinier, A. and G. Fournet, Small-angle scattering of X-rays. Structure of matter series. 1955, New York,: Wiley. $268 \mathrm{p}$.

66. Bunk, O., et al., Multimodal x-ray scatter imaging. New Journal of Physics, 2009. 11.

67. Gourrier, A., et al., Artificially Heated Bone at Low Temperatures: A Quantitative Scanning-small-angle X-Ray Scattering Imaging Study of the Mineral Particle Size. Archéosciences, 2012, in press.

68. Roschger, P., et al., Bone mineralization density distribution in health and disease. Bone, 2008. 42(3): p. 456-466.

69. Reiche, I. and A. Gourrier, 2012, in preparation.

70. Ando, M., et al., X-ray dark-field imaging and its application to medicine. Radiation Physics and Chemistry, 2004. 71: p. 899-904. 
71. Diemoz, P.C., et al., A simplified approach for computed tomography with an X-ray grating interferometer. Optics Express, 2011. 19(3): p. 1691-1698. 